Meta

Journal des traducteurs

Translators' Journal

\title{
Dancette, Jeanne et Christophe Réthoré (2000) : Dictionnaire analytique de la distribution/Analytical Dictionary of Retailing, Les Presses de l'Université de Montréal, 380 pages.
}

\section{Alain Duval}

Volume 47, numéro 1, mars 2002

URI : https://id.erudit.org/iderudit/007998ar

DOI : https://doi.org/10.7202/007998ar

Aller au sommaire du numéro

Éditeur(s)

Les Presses de l'Université de Montréal

ISSN

0026-0452 (imprimé)

1492-1421 (numérique)

Découvrir la revue

Citer ce compte rendu

Duval, A. (2002). Compte rendu de [Dancette, Jeanne et Christophe Réthoré (2000) : Dictionnaire analytique de la distribution/Analytical Dictionary of Retailing, Les Presses de l'Université de Montréal, 380 pages.] Meta, 47(1),

129-130. https://doi.org/10.7202/007998ar d'utilisation que vous pouvez consulter en ligne.

https://apropos.erudit.org/fr/usagers/politique-dutilisation/ 
L'or de l'Abbé retrace la demande de Pierre le Vénérable, abbé de Cluny, en 1142 pour une traduction en latin du Coran. Il est probable que la traduction visait la conversion des «infidèles", mais la question reste: pourquoi traduire un texte en latin alors que les destinataires ignoraient cette langue? L'École de traduction de Tolède a-t-elle vraiment existé ou est-ce une création due à une interprétation mal orientée de textes? Les traductions faites après 1250 de l'arabe en castillan, ou du castillan en latin et en français, ont contribué à la gloire d'Alphonse $\mathrm{X}$ assurant le développement économique et linguistique du royaume. Si Bagdad s'est développée comme centre de traduction au neuvième siècle, c'est sans doute dû à la présence de nombreux traducteurs, mais il faut également tenir compte du développement local de l'industrie du papier. L'histoire semble se répéter d'une certaine façon en Castille, où les traductions commanditées par Alphonse X profitent de la création de la même accessibilité au papier. La traduction de l'Ancien Testament en castillan par le rabbin Mose Arragel donne lieu à la Bible de Alba, un document très hybride comprenant des gloses rabbiniques et chrétiennes. C'est également au quinzième siècle que la morphologie de l'espagnol modifie les composés à racine translad- (transladador, transladation) en traduc- (traductor, traducciòn). Le changement n'est pas simplement morphologique: il y a également une transformation quant à la hiérarchie supposée des langues. Tout change, si Christophe Colomb quitte l'Espagne à la recherche du Nouveau Monde, le 3 août 1492, la veille dix navires partent de Barcelone chargés de juifs expulsés! La découverte du Nouveau Monde amème le débat sur le statut les langues amérindiennes et la création de l'empire linguistique castillan. La ContreRéforme crée un autre exil et accentue le triomphe du castillan en même temps qu'un certain repli culturel et une distanciation du mouvement traductionnel humaniste de l'Europe du Nord. L'expérience des Jeux olympiques de Barcelone avec quatre langues officielles permet également une meilleure compréhension de certaines réalités et la déduction d'enseignements pratiques utiles. L'époque moderne n'est pas ignorée et le problème de la mondialisation impose des réformes de l'enseignement de la traduction, mais aussi la discussion et la remise en cause de certains points de vue indispensables pour mieux percevoir les exigences de l'avenir.

C'est sans nul doute un livre plaisant à lire et qui provoque la réflexion et la comparaison avec d'autres situations. Sa lecture est donc à recommander. C'est d'un certain point de vue et, pour certains aspects, un complément particularisé au livre de Scott L. Montgomery: Science in Translation. Movements of Knowledge Through Cultures and Times.

André Clas

Université de Montréal, Montréal, Canada

Dancette, Jeanne et Christophe Réthoré (2000) : Dictionnaire analytique de la distribution/Analytical Dictionary of Retailing, Les Presses de l'Université de Montréal, 380 pages.

Comment joindre l'utile à l'original? Le travail réalisé par Jeanne Dancette et Christophe Réthoré en donne un excellent exemple: ni vraiment dictionnaire bilingue, ni austère manuel de cours, leur ouvrage présente de manière neuve et dynamique un état actuel du lexique de la distribution en français et en anglais. 
Il convient tout d'abord de noter l'exhaustivité et la pertinence du corpus. Les glossaires traditionnels, étant donné les limites restreintes du champ envisagé, conduisent trop souvent les auteurs à dévier de leur propos pour rassembler sans critères de sélection clairement définis des éléments aléatoirement puisés dans des domaines connexes plus ou moins pertinents. Rien de tel ici. La couverture impressionnante (3500 termes dans chaque langue) est remarquablement ciblée: "acheteur en ligne, entreprise franchisante, zone de chalandise» ne sont là que quelques exemples qui montrent la rigueur et l'efficacité des choix, authentifiés dans les articles par une zone de contextes composée de citations en français et en anglais choisies dans des revues et des ouvrages récents.

Mais, au-delà de la simple couverture lexicale, c'est dans le rapport très original qui est entretenu entre langue source et langue cible que réside la véritable utilité de l'ouvrage. Les vedettes sont en anglais. Elles sont accompagnées de leurs synonymes ou quasi-synonymes usuels, et naturellement de leurs traductions, là aussi hiérarchisées en traduction principale et traductions secondaires. Cette information est déjà fort complète et beaucoup de dictionnaires traditionnels s'arrêteraient là. Pourtant, ici, c'est seulement alors que l'article commence véritablement: plusieurs paragraphes rédigés en français, précédés chacun d'un titre à la typographie nettement différenciée, présentent d'abord une définition détaillée du mot vedette, puis des précisions sémantiques et enfin un inventaire de relations internotionnelles et des compléments d'information expliquant les similitudes et les différences que le concept exprimé par la vedette entretient avec les concepts qui lui sont liés. L'entrée traduite de départ s'ouvre ainsi sur une véritable monographie qui structure et développe le thème.

Dans la mesure où les entrées sont en anglais et le corps des articles en français, s'agit-il d'un dictionnaire bilingue à l'usage des francophones désirant décoder le vocabulaire anglais de la distribution? Cette vision serait extrêmement réductrice car les anglophones comme les francophones y trouvent leur compte dans la mesure où tous les mots opérants du corps de l'article en français sont dûment étiquetés de leur la traduction anglaise adéquate placée entre parenthèses. Un second niveau d'équivalents bilingues est par là même constitué. Au-delà du simple mot vedette, le francophone acquiert ainsi dynamiquement en anglais toutes les connaissances lexicales du réseau notionnel concerné alors que l'anglophone part à la découverte du français à travers les mises en situation naturelles de la partie didactique.

Des regrets? Bien sûr, mais ils sont le pendant nécessaire des qualités de ce dictionnaire et en particulier de son approche novatrice des rapports traductifs et de sa volonté d'analyse exhaustive du domaine. Ainsi le traducteur pressé ne trouvera peut-être pas aussi rapidement qu'il le voudrait les équivalents qu'il cherche à partir du double index bilingue de la fin. Cet ouvrage n'est pas un glossaire superficiel conçu pour une consultation rapide. C'est un livre solide et sérieux dont les articles demandent l'attention et le temps nécessaires à la connaissance approfondie de chaque concept étudié. 